\title{
IncRNA DGCR5 inhibits the proliferation of colorectal cancer cells by downregulating miR-21
}

\author{
HANSHENG HUANG ${ }^{1}$, XIAOMEI YANG ${ }^{1}$, JUN CHEN ${ }^{2}$, JUNMIN FU ${ }^{1}$, \\ CHAOTING CHEN $^{1}$, JIANMEI WEN ${ }^{1}$ and QIAO MO ${ }^{1}$
}

\begin{abstract}
${ }^{1}$ Department of Medical Oncology, The Second Affiliated Hospital of Guangxi University of Science and Technology, Liuzhou, Guangxi 545006; ${ }^{2}$ Department of Pathology, The Affiliated Tumor Hospital of Guangxi Medical University, Nanning, Guangxi 530021, P.R. China
\end{abstract}

Received February 5, 2019; Accepted June 13, 2019

DOI: $10.3892 / 01.2019 .10671$

\begin{abstract}
Long non-coding RNA (lncRNA) DiGeorge syndrome critical region gene 5 (DGCR5) serves roles as a tumor suppressor or oncogene in different types of cancer. The current study aimed to explore the role of DGCR5 in colorectal cancer (CRC). It was revealed that the expression of DGCR5 was downregulated, while microRNA (miR)-21 was upregulated in CRC. The expression level of DGCR5 in tumor tissue decreased, while expression levels of miR-21 increased, with advancing stages of the disease. The expression levels of DGCR5 and miR-21 were inversely associated in tumor tissues. In CRC cells in vitro, miR-21 overexpression failed to significantly affect DGCR5, while DGCR5 overexpression resulted in reduced expression levels of miR-21. DGCR5 overexpression showed no significant effects on cancer cell migration and invasion, but suppressed cancer cell proliferation in vitro. miR-21 overexpression increased cancer cell proliferation and attenuated the effects of DGCR5 overexpression. Therefore, lncRNA DGCR5 may inhibit the proliferation of CRC cells by downregulating miR-21.
\end{abstract}

\section{Introduction}

Incidence of colorectal cancer (CRC) was the third most common type of cancer in certain regions of the world in 2018, such as the USA (1). CRC is a fatal disease, resulting in $>500,000$ mortalities every year worldwide (2). Patients with early stages of CRC are treated surgically, followed by adjuvant therapy to inhibit cancer cell proliferation and metastasis (3). However, a considerable number of patients with $\mathrm{CRC}$ are diagnosed with metastasis to the lymph nodes and

Correspondence to: Dr Xiaomei Yang, Department of Medical Oncology, The Second Affiliated Hospital of Guangxi University of Science and Technology, 17 Jian Pan Road, Liuzhou, Guangxi 545006, P.R. China

E-mail: ed01873@163.com

Key words: colorectal cancer, long non-coding RNA DiGeorge syndrome critical region gene 5 , microRNA-21 major organs, such as the lungs and liver, leading to poor treatment outcomes and a low overall 5 -year survival rate $(4,5)$. Therefore, novel therapeutic targets are required to improve the survival of patients with advanced CRC.

Genome-wide analysis of genetic alterations has revealed that certain somatic and germline mutations are critical determinants of tumorigenesis in CRC $(6,7)$. Long non-coding RNAs (lncRNAs) are RNA transcripts $>200$ nucleotides in length with non-protein-coding abilities, and have been identified as critical players in cancer biology through their regulatory roles in gene expression at posttranscriptional or translational levels and through epigenetic pathways (8). In effect, certain lncRNAs are potential therapeutic targets for the treatment of many types of cancers, such as lung cancer, liver cancer and CRC $(9,10)$. However, the application of most lncRNAs in cancer therapies is limited by their unknown functions. IncRNA DiGeorge syndrome critical region gene 5 (DGCR5) has been implicated in many types of cancer, and serves a cancer-specific role as a tumor suppressor in papillary thyroid carcinoma or as an oncogene lung adenocarcinoma $(11,12)$. The majority of studies investigating the role of DGCR5 in cancer biology have revealed its interactions with microRNAs (miRNAs/miRs), a group of small non-coding RNA molecules $\sim 22$ nucleotides in length which serve important roles in gene expression regulation through the cleavage of transcripts $(11,12)$. miR-21 is a well-characterized oncogenic miRNA in several types of cancer, including CRC (13). miR-21 participates in CRC by regulating various cancer cell behaviors through the interaction with multiple downstream tumor suppressive and oncogenic pathways, such as the phosphatase and tensin homolog (PTEN) and signal transducer and activator of transcription 3 pathways, respectively (13). Preliminary data has revealed that DGCR5 and miR-21 were inversely correlated in human CRC tissues (data not shown). The present study investigated the role of DGCR5 in CRC as well as the possible interaction between DGCR5 and miR-21.

\section{Materials and methods}

Patients. The present study included 65 patients with CRC enrolled at The Second Affiliated Hospital of Guangxi University of Science and Technology between March 2016 and 
March 2018. The patients included 35 males and 30 females and their mean age was 51.3 \pm 5.7 (range, 34-66 years). The diagnosis of the patients was confirmed by histopathological examinations. The inclusion criteria for participation in the current study were as follows: i) patients diagnosed with CRC for the first time; ii) patients had not been treated prior to admission; and iii) patients with complete medical records. The exclusion criteria were as follows: i) patients with previous history of malignancy; ii) patients who received treatment within 3 months prior to admission; iii) patients who were transferred from other hospitals. Based on the American Joint Committee on Cancer staging criteria (AJCC) used (14), there were 14, 20, 18 and 13 patients at stage I, II, III and IV, respectively. The Ethics Committee of The Second Affiliated Hospital of Guangxi University of Science and Technology approved the current study and all patients signed informed consent.

Local blast. A local blast analysis was performed using NCBI-blast software (version 2.2.30; National Center for Biotechnology Information). In this analysis, human miRNAs sequences (http://www.mirbase.org) were used as queries, and DGCR5 was used as a targeted database.

Specimens and cell lines. A biopsy was performed on each patient to obtain cancer and adjacent healthy tissues $(2 \mathrm{~cm}$ distance from the tumor site) from each patient. The weight of the tissues ranged between 0.07 and $0.11 \mathrm{~g}$. Tissues were stored at $-70^{\circ} \mathrm{C}$ until use. All tissue samples were confirmed by histopathological examination. RKO (American Type Culture Collection) and CR4 (Sigma-Aldrich; Merck KGaA) human $\mathrm{CRC}$ cell lines were used to perform in vitro cell experiments. Cells were cultured according to the manufacturer's protocol using Eagle's Minimum Essential Medium (Sigma-Aldrich; Merck KGaA) supplemented with $10 \%$ fetal bovine serum (FBS; Sigma-Aldrich; Merck KGaA) at $5 \% \mathrm{CO}_{2}$ and $37^{\circ} \mathrm{C}$.

Total RNA extraction and reverse-transcription quantitative polymerase chain reaction $(R T-q P C R)$. For the analysis of DGCR5 expression levels, RNAzol ${ }^{\circledR}$ RT reagent (Sigma-Aldrich; Merck KGaA) was used to extract total the RNA from CRC and adjacent healthy tissues as well as the RKO and CR4 cell lines. RNA samples were reverse transcribed into cDNA using the AMV Reverse Transcriptase kit (Promega Corporation) at the following thermal conditions: $15 \mathrm{~min}$ at $55^{\circ} \mathrm{C}$ and $10 \mathrm{~min}$ at $80^{\circ} \mathrm{C}$. qPCR was subsequently performed using the $\mathrm{SYBR}{ }^{\circledR}$ Green master mix (Bio-Rad Laboratories, Inc.) using 18S rRNA as an endogenous control. The following primer sequences were used: DGCR5 forward, 5'-CCAAGCCTGTCTGTGTGT TC-3' and reverse, 5'-GGGAGACACAGACCACAAGA-3'; and $18 \mathrm{~S}$ forward, 5'-GCTTAATTTGACTCAACACGGGA-3' and reverse, 5'-AGCTATCAATCTGTCAATCCTGTC-3'. Thermal conditions were: $1 \mathrm{~min}$ at $95^{\circ} \mathrm{C}$, followed by 40 cycles of $10 \mathrm{sec}$ at $95^{\circ} \mathrm{C}$ and $40 \mathrm{sec}$ at $95^{\circ} \mathrm{C}$. For the analysis of miR-21 expression levels, extraction of miRNAs from CRC and adjacent healthy tissues as well as the RKO and CR4 cell lines was performed using a microRNA purification kit (cat. no. 21300; Norgen Biotek Corp.) according to manufacturer's protocol. TaqMan microRNA Reverse Transcription kit (Thermo Fisher Scientific, Inc.) was used to perform reverse transcription according to manufacturer's protocol. TaqMan ${ }^{\mathrm{TM}}$ Fast Advanced Master mix
(Thermo Fisher Scientific, Inc.) was used to prepare all qPCR reaction mixtures using U6 as the endogenous control. The following primer was used for the reaction: miR-21 forward, 5'-GCCCGCTAGCTTATCAGACTGATG-3'. The miR-21 reverse primer and U6 forward and reverse primers were included in the TaqMan ${ }^{\mathrm{TM}}$ Fast Advanced Master mix kit. The therrmal conditions were: $1 \mathrm{~min}$ at $95^{\circ} \mathrm{C}$, followed by 40 cycles of $10 \mathrm{sec}$ at $95^{\circ} \mathrm{C}$ and $20 \mathrm{sec}$ at $95^{\circ} \mathrm{C}$. All RT-qPCR reactions were performed in triplicate.

All data were analyzed using the $2^{-\Delta \Delta C} q$ method (15) and each experiment was performed in triplicate.

Transient transfection. Sequences for the negative control (5'-CGCGCUUCGGUUUAACUAGC-3') miRNA and miR-21 mimic (5'-CAACACCAGUCGAUGGGCUGU-3') were obtained from Sigma-Aldrich; Merck KGaA. The DGCR5-expression pcDNA3.1 and empty pcDNA3.1 vectors were obtained from by Sangon Biotech Co., Ltd. A total of $10^{5} \mathrm{CRC}$ or RKO cells were transfected with $40 \mathrm{nM}$ miRNA or $10 \mathrm{nM}$ vector using Lipofectamine ${ }^{\circledR} 2000$ (Thermo Fisher Scientific, Inc.) according to manufacturer's protocol. Two types of control cells, including cells without transfection (C) and cells transfected with empty vectors or negative control miRNAs (NC) were included. Subsequent experiments were performed $24 \mathrm{~h}$ following transfection.

Cell proliferation assay. CRC and RKO cells were harvested $24 \mathrm{~h}$ following transfection and single cell suspensions were prepared using Eagle's Minimum Essential Medium supplemented with $10 \%$ FBS to a final cell density of $4 \times 10^{4}$ cell $/ \mathrm{ml}$. Cells suspensions were cultured at $37^{\circ} \mathrm{C}$ and $5 \% \mathrm{CO}_{2}$ in a 96 -well plate containing $0.1 \mathrm{ml}$ per well. A total of $10 \mu \mathrm{l}$ Cell Counting Kit-8 solution (Sigma-Aldrich; Merck KGaA) was added into each well every $24 \mathrm{~h}$ for $96 \mathrm{~h}$. The optical density was measured at a wavelength of $450 \mathrm{~nm}$ to represent cell proliferation.

Statistical analysis. Three biological replicates were included in each experiment. Differences between CRC and healthy adjacent tissues were analyzed by the paired t-test. Differences among different clinical stages and cell transfection groups were analyzed by the one-way ANOVA and Tukey test. Linear regression was performed to analyze the association between the expression levels of DGCR5 and miR-21 in CRC and healthy adjacent tissues. $\mathrm{P}<0.05$ was considered to indicate a statistically significant difference.

\section{Results}

DGCR5 and miR-21 are dysregulated in CRC tissues. The expression levels of DGCR5 and miR-21 in CRC and healthy adjacent tissues were quantified by RT-qPCR and the expression data were analyzed using the paired t-test. It was observed that DGCR5 was significantly downregulated (Fig. 1A), while miR-21 was significantly upregulated (Fig. 1B), in CRC tissues compared with healthy adjacent tissues in patients with CRC $(\mathrm{P}<0.05)$, indicating the involvement of DGCR5 and miR-21 in CRC.

Expression levels of DGCR5 and miR-21 in CRC tissues are affected by clinical stages. The expression levels of DGCR5 

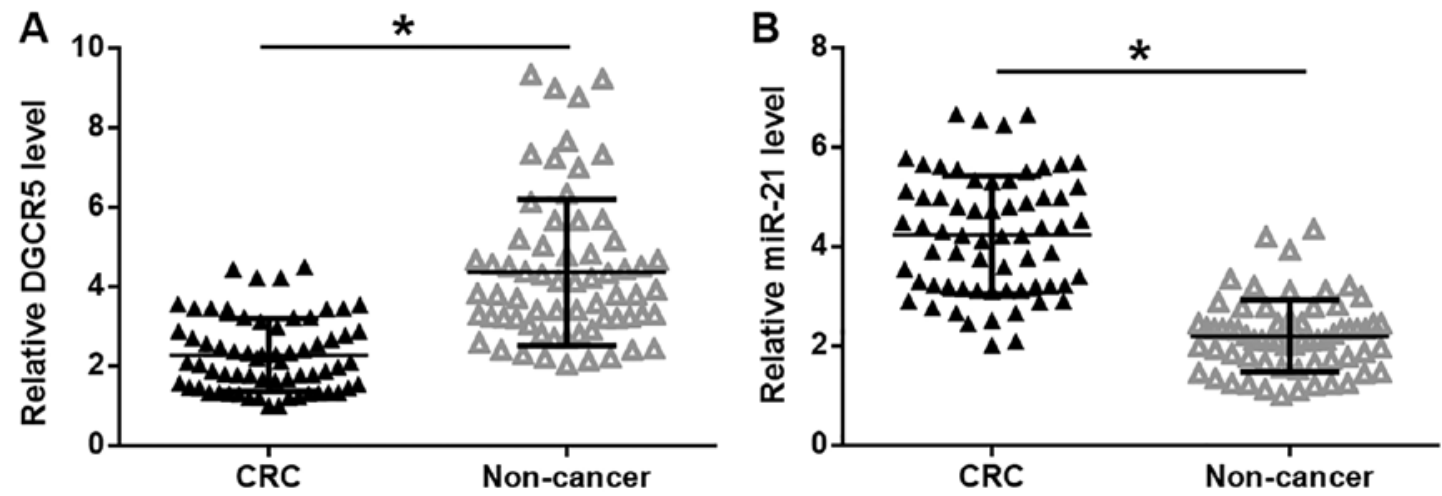

Figure 1. DGCR5 and miR-21 were dysregulated in CRC tissues. Analysis of reverse-transcription quantitative polymerase chain reaction data using the paired t-test revealed that (A) DGCR5 was significantly downregulated while (B) miR-21 was significantly upregulated in CRC tissue compared with adjacent non-cancer tissue in patients with CRC. " $\mathrm{P}<0.05$, as indicated. DGCR5, DiGeorge syndrome critical region gene 5; miR, microRNA; CRC, colorectal cancer.
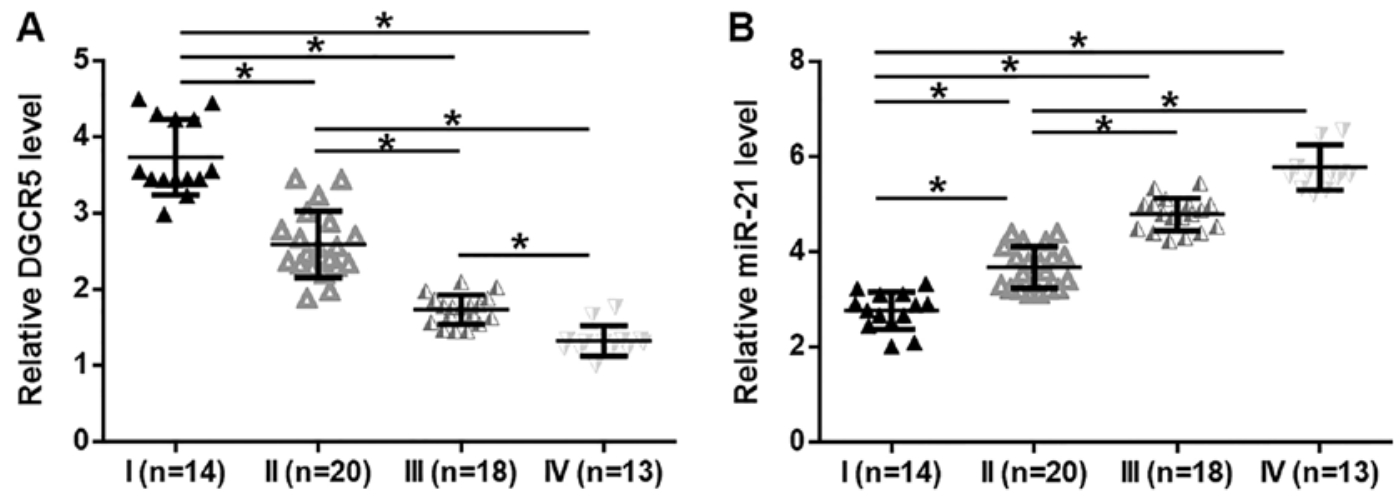

Figure 2. Expression levels of DGCR5 and miR-21 in CRC tissues were affected by clinical stages. Differences in expression levels of DGCR5 and miR-21 among patients with different clinical stages were analyzed using the one-way analysis of variance followed by the Tukey post hoc test. (A) DGCR5 expression levels decreased while (B) miR-21 expression levels increased with advanced clinical stages. ${ }^{*} \mathrm{P}<0.05$, as indicated. DGCR5, DiGeorge syndrome critical region gene 5; miR, microRNA; CRC, colorectal cancer.

and miR-21 in patients with different clinical stages of CRC were analyzed using the one-way ANOVA and Tukey test. The expression levels of DGCR5 in CRC tissues were significantly decreased (Fig. 2A), while expression levels of miR-21 were significantly increased (Fig. 2B), with advancing clinical stages $(\mathrm{P}<0.05)$.

DGCR5 and miR-21 expression levels are inversely associated in CRC tissues. Linear regression was performed to analyze the association between the expression levels of DGCR5 and miR-21. It was observed that DGCR5 and miR-21 were inversely associated in CRC tissues (Fig. 3A). However, the association between DGCR5 and miR-21 was not statistically significant in healthy adjacent tissues (Fig. 3B). It suggested that DGCR5 and miR-21 may interact with each other in CRC.

DGCR5 overexpression causes the downregulation of $m i R-21$ in CRC cells. In order to explore the interaction between DGCR5 and miR-21 in CRC, a DGCR5 expression vector and miR-21 mimic were transfected into CRC and RKO cell lines. Compared with control (C) and negative control (NC) groups, expression levels of DGCR5 and miR-21 were upregulated by more than 2 fold at $24 \mathrm{~h}$ after transfection (Fig. 4A; $\mathrm{P}<0.05$ ), indicating the successful transfections. In addition, miR-21 overexpression failed to significantly affect DGCR5 (Fig. 4B), while DGCR5 overexpression led to reduced expression levels of miR-21 (Fig. 4C; $\mathrm{P}<0.05$ ).

DGCR5 regulates cancer cell proliferation but not migration and invasion through miR-21. Comparing with the $\mathrm{C}$ and $\mathrm{NC}$ groups, DGCR5 overexpression showed no significant effects on RKO and CR4 cell migration and invasion (data not shown; revealed by Transwell migration and invasion assay). By contrast, DGCR5 overexpression resulted in inhibited RKO and $\mathrm{CR} 4$ cell proliferation compared with the $\mathrm{C}$ and $\mathrm{NC}$ groups (Fig. 5; $\mathrm{P}<0.05$ ). miR-21 overexpression resulted in increased cancer cell proliferation and attenuated the effects of DGCR5 overexpression (Fig. 5; P<0.05).

\section{Discussion}

lncRNA DGCR5 plays a role as tumor suppressor in certain types of cancer, including lung cancer, but serves as an oncogene in other types of cancer, including papillary thyroid carcinoma and liver cancer $(11,12,16)$. The present study revealed the tumor suppressive role of DGCR5 in CRC and demonstrated that DGCR5 may inhibit the proliferation of CRC cells by downregulating miR-21, an oncogenic miRNA in CRC (13). 

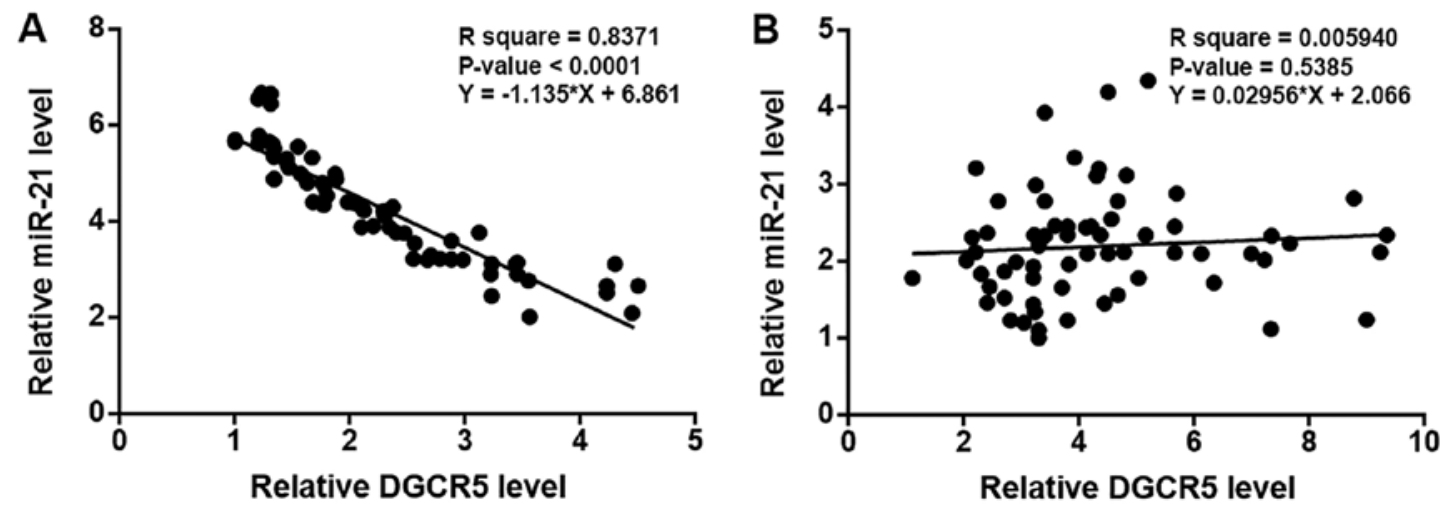

Figure 3. DGCR5 and miR-21 were inversely associated in CRC tissues. Linear regression analysis showed that DGCR5 and miR-21 were (A) inversely associated in CRC tissues but not in (B) adjacent non-cancer tissues. DGCR5, DiGeorge syndrome critical region gene 5; miR, microRNA; CRC, colorectal cancer.
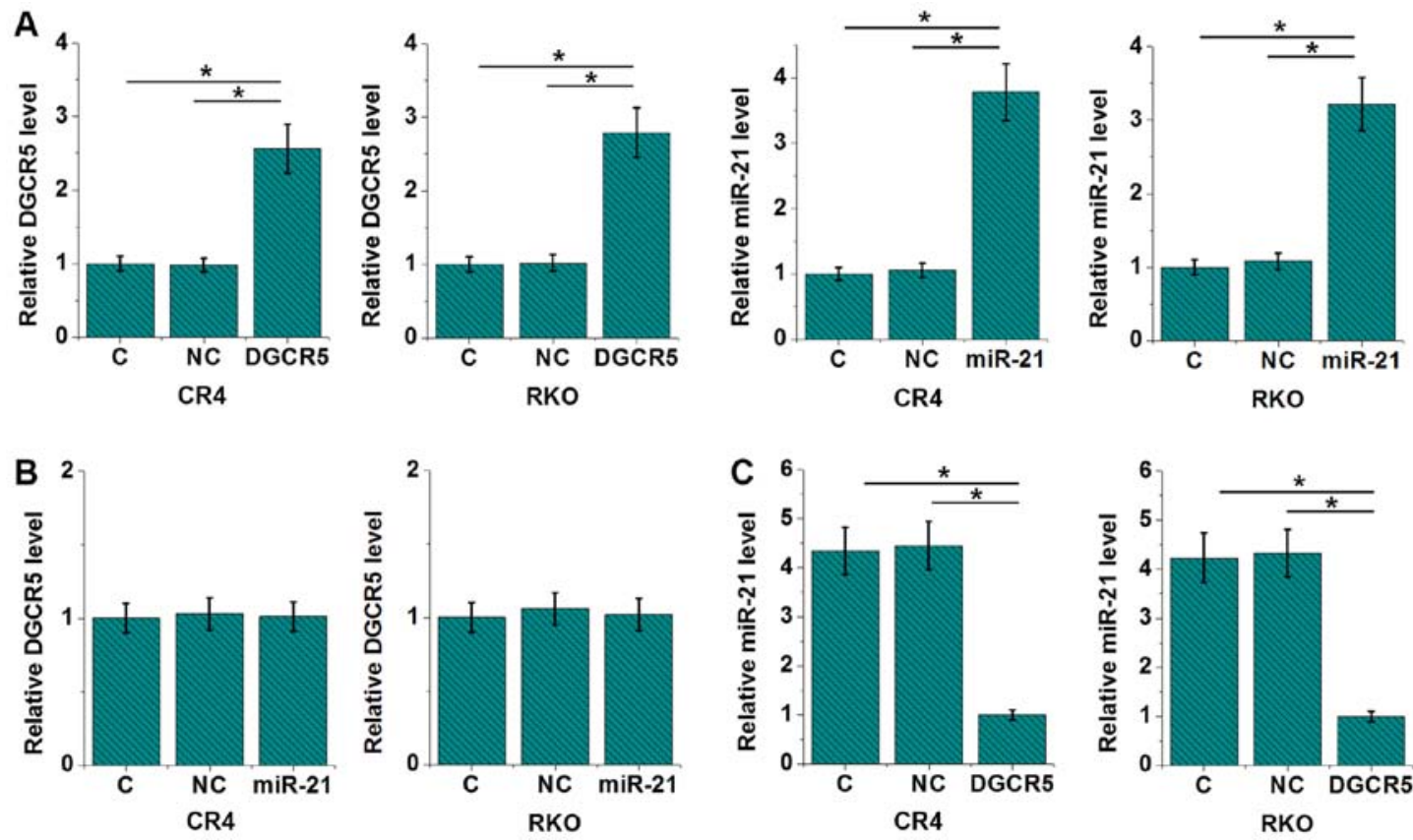

Figure 4. DGCR5 overexpression downregulated miR-21 expression in CRC cell lines. (A) Compared with the C and NC groups, expression levels of DGCR5 and miR-21 were upregulated $>2$-fold $24 \mathrm{~h}$ following transfection. (B) miR-21 overexpression failed to significantly affect DGCR5 expression and (C) DGCR5 overexpression reduced expression levels of miR-21. "P<0.05, as indicated. DGCR5, DiGeorge syndrome critical region gene 5; miR, microRNA; CRC, colorectal cancer; C, control; NC, negative control.
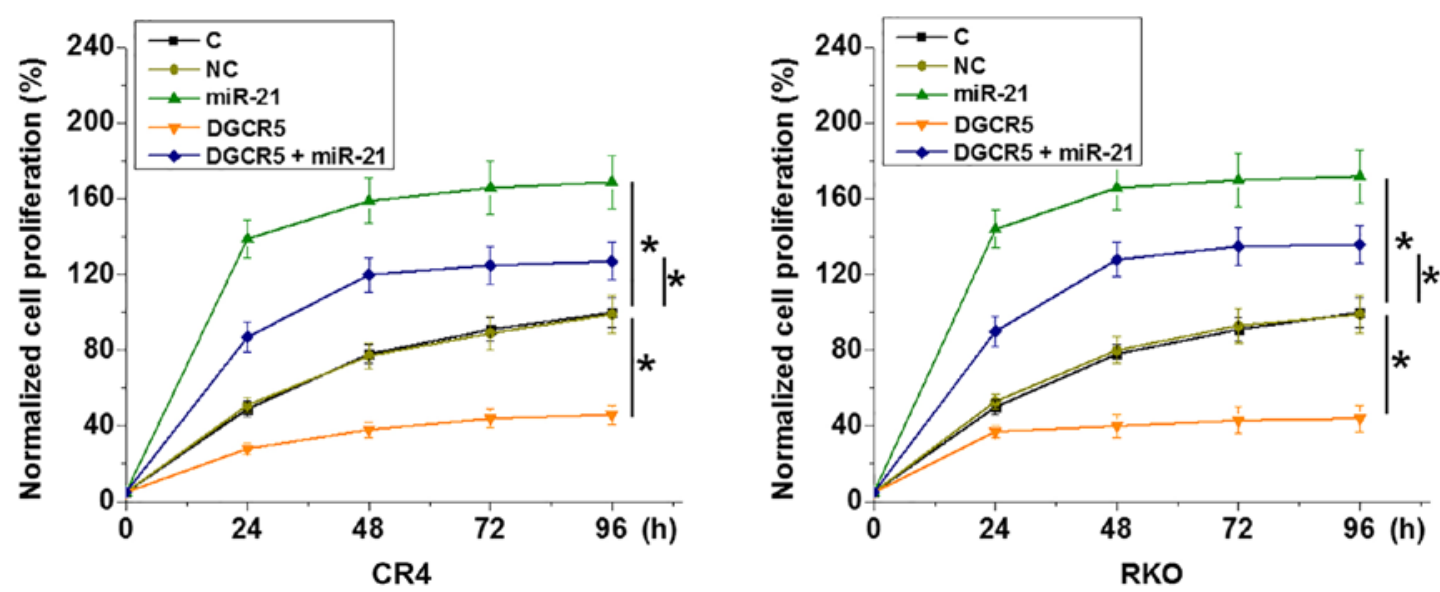

Figure 5. DGCR5 regulated cancer cell proliferation through miR-21. DGCR5 overexpression inhibited cancer cell proliferation. miR-21 overexpression promoted cancer cell proliferation and attenuated the effects of DGCR5 overexpression. " $\mathrm{P}<0.05$, as indicated. DGCR5, DiGeorge syndrome critical region gene 5; miR, microRNA; C, control; NC, negative control. 
DGCR5 has opposite functions in different types of cancer. DGCR5 was upregulated during the development of lung cancer, and DGCR5 overexpression inhibited hsa-mir-22-3p to promote cancer development (11). By contrast, DGCR5 inhibits miR-2861 to serve a tumor suppressive role in papillary thyroid carcinoma (12). The present study revealed that that DGCR5 was downregulated in CRC tissues compared with healthy adjacent non-cancer in patients with CRC patients. Additionally, overexpression of DGCR5 decreased the proliferation of CRC cells in vitro. Therefore, the results obtained in the present study suggested that DGCR5 serves a tumor suppressive role in CRC.

To the best of our knowledge, the present study was the first to report the inhibitory role of DGCR5 on miR-21, which as a well-characterized oncogenic miRNA in several types of cancer (17). miR-21 expression in cancer development may be regulated by certain lncRNAs $(18,19)$. One possible mechanism of the interaction between IncRNAs and miRNAs is that lncRNAs may sponge miRNAs and inhibit their function (20). However, a BLAST (NCBI) search did not reveal potential binding sites for miR-21 on DGCR5, suggesting that other molecular mechanisms may mediate interactions between lncRNAs and miRNAs. Consistently, preliminary luciferase assay data (data not shown) suggested an indirect interaction between miR-21 and DGCR5. The relative luciferase signals of miR-21 and negative control were $100 \pm 5.6$ vs. $99.7 \pm 4$.9. It has been shown that both DGCR5 and miR-21 interact with PTEN $(21,22)$. In addition, PTEN is downregulated in CRC (23), and PTEN can mediate the interaction between certain IncRNAs and miRNAs (24). Therefore, PTEN may mediate the interaction between DGCR5 and miR-21; however, future studies are required to elucidate the molecular mechanism involved.

DGCR5 overexpression resulted in downregulated miR-21 but failed to significantly affect CRC cell migration and invasion (data not shown). miR-21 may regulate in vitro CRC cell invasion and migration (16). Therefore, DGCR5 may interact with multiple effectors to regulate CRC cell behavior. Future studies are required to identify the effectors involved.

In conclusion, DGCR5 was downregulated in CRC tissues compared to healthy adjacent samples in patients with CRC and overexpression of DGCR5 in vitro inhibited CRC cell proliferation by downregulating miR-21.

\section{Acknowledgements}

Not applicable.

\section{Funding}

No funding was received.

\section{Availability of data and materials}

The datasets used and/or analyzed during the present study are available from the corresponding author upon reasonable request.

\section{Authors' contributions}

$\mathrm{HH}$ and $\mathrm{XY}$ designed the experiments. HH, XY, JC and JF performed the experiments. CC, JW and QM collected and analyzed the data. XY drafted the manuscript. All authors have read and approved this manuscript.

\section{Ethics approval and consent to participate}

The Ethics Committee of The Second Affiliated Hospital of Guangxi University of Science and Technology approved the current study and all patients signed informed consent.

\section{Patient consent for publication}

Not applicable.

\section{Competing interests}

The authors declare that they have no competing interests.

\section{References}

1. Siegel RL, Miller KD and Jemal A: Cancer statistics, 2018. CA Cancer J Clin 68: 7-30, 2018.

2. Haggar FA and Boushey RP: Colorectal cancer epidemiology: Incidence, mortality, survival and risk factors. Clin Colon Rectal Surg 22: 191-197, 2009.

3. Van Cutsem E, Lenz HJ, Köhne CH, Heinemann V, Tejpar S, Melezínek I, Beier F, Stroh C, Rougier P, van Krieken JH and Ciardiello F: Fluorouracil, leucovorin, and irinotecan plus cetuximab treatment and RAS mutations in colorectal cancer. J Clin Oncol 33: 692-700, 2015.

4. Zhang M, Miao F, Huang R, Liu W, Zhao Y, Jiao T, Lu Y, Wu F, Wang $\mathrm{X}$, Wang $\mathrm{H}$, et al: RHBDD1 promotes colorectal cancer metastasis through the Wnt signaling pathway and its downstream target ZEB1. J Exp Clin Cancer Res 37: 22, 2018.

5. Marquez J,Fernandez-Piñeiro I, Araúzo-Bravo MJ,Poschmann G, Stühler K, Khatib AM, Sanchez A, Unda F, Ibarretxe G, Bernales I and Badiola I: Targeting liver sinusoidal endothelial cells with miR-20a-loaded nanoparticles reduces murine colon cancer metastasis to the liver. Int J Cancer 143: 709-719, 2018.

6. Fearon ER and Vogelstein B: A genetic model for colorectal tumorigenesis. Cell 61: 759-767, 1990.

7. Sillars-Hardebol AH, Carvalho B, de Wit M, Postma C, Delis-van Diemen PM, Mongera S, Ylstra B, van de Wiel MA, Meijer GA and Fijneman RJ: Identification of key genes for carcinogenic pathways associated with colorectal adenoma-to-carcinoma progression. Tumour Biol 31: 89-96, 2010.

8. Gutschner T and Diederichs S: The hallmarks of cancer: A long non-coding RNA point of view. RNA Biol 9: 703-719, 2012.

9. Spizzo R, Almeida MI, Colombatti A and Calin GA: Long non-coding RNAs and cancer: A new frontier of translational research? Oncogene 31: 4577-4587, 2012.

10. Wahlestedt C: Targeting long non-coding RNA to therapeutically upregulate gene expression. Nat Rev Drug Discov 12:433-446, 2013.

11. Dong HX, Wang R, Jin XY, Zeng J and Pan J: LncRNA DGCR5 promotes lung adenocarcinoma (LUAD) progression via inhibiting hsa-mir 22-3p. J Cell Physiol 233: 4126-4136, 2018.

12. Chen F, Yin S, Zhu J, Liu P, Yang C, Feng Z and Deng Z: IncRNA DGCR5 acts as a tumor suppressor in papillary thyroid carcinoma via sequestering miR-2861. Exp Ther Med 17: 895-900, 2019.

13. Feiersinger F, Nolte E, Wach S, Rau TT, Vassos N, Geppert C, Konrad A, Merkel S, Taubert H, Stürzl M and Croner RS: MiRNA-21 expression decreases from primary tumors to liver metastases in colorectal carcinoma. PLoS One 11: e0148580, 2016.

14. Hari D M, Leung AM, Lee JH, Sim MS, Vuong B, Chiu CG and Bilchik AJ: AJCC Cancer Staging Manual 7th edition criteria for colon cancer: Do the complex modifications improve prognostic assessment? J Am Coll Surg 217: 181-190, 2013.

15. Livak KJ and Schmittgen TD: Analysis of relative gene expression data using real-time quantitative PCR and the 2(-Delta Delta C(T)) method. Methods 25: 402-408, 2001.

16. Huang R, Wang X, Zhang W, Zhangyuan G, Jin K, Yu W, Xie Y, $\mathrm{Xu}$ X, Wang H and Sun B: Down-regulation of LncRNA DGCR5 correlates with poor prognosis in hepatocellular carcinoma. Cell Physiol Biochem 40: 707-715, 2016. 
17. Pan X, Wang ZX and Wang R: MicroRNA-21: A novel therapeutic target in human cancer. Cancer Biol Ther 10: 1224-1232, 2010.

18. Hu L, Ye H, Huang G, Luo F, Liu Y, Liu Y, Yang X, Shen J, Liu Q and Zhang J: Long noncoding RNA GAS5 suppresses the migration and invasion of hepatocellular carcinoma cells via miR-21. Tumuor Biol 37: 2691-2702, 2016.

19. Ma MZ, Chu BF, Zhang Y, Weng MZ, Qin YY, Gong W and Quan ZW: Long non-coding RNA CCAT1 promotes gallbladder cancer development via negative modulation of miRNA-218-5p. Cell Death Dis 6: e1583, 2015.

20. Zhou Q, Chen F, Zhao J, Li B, Liang Y, Pan W, Zhang S, Wang X and Zheng D: Long non-coding RNA PVT1 promotes osteosarcoma development by acting as a molecular sponge to regulate miR-195. Oncotarget 7: 82620-82633, 2016.

21. Xu Y, Zhang G, Zou C, Gong Z, Wang S, Liu J, Ma G, Liu X, Zhang $\mathrm{W}$ and Jiang P: Long noncoding RNA DGCR5 suppresses gastric cancer progression by acting as a competing endogenous RNA of PTEN and BTG1. J Cell Physiol 234: 11999-12010, 2019.
22. Song L, Liu S, Zhang L, Yao H, Gao F, Xu D and Li Q: MiR-21 modulates radiosensitivity of cervical cancer through inhibiting autophagy via the PTEN/Akt/HIF-1 $\alpha$ feedback loop and the Akt-mTOR signaling pathway. Tumuor Biol 37: 12161-12168, 2016.

23. Rana C, Piplani H, Vaish V, Nehru B and Sanyal SN: Downregulation of PI3-K/Akt/PTEN pathway and activation of mitochondrial intrinsic apoptosis by Diclofenac and Curcumin in colon cancer. Mol Cell Biochem 402: 225-241, 2015.

24. Yang N, Chen J, Zhang H, Wang X, Yao H, Peng Y and Zhang W: LncRNA OIP5-AS1 loss-induced microRNA-410 accumulation regulates cell proliferation and apoptosis by targeting KLF10 via activating PTEN/PI3K/AKT pathway in multiple myeloma. Cell Death Dis 8: e2975, 2017. 